\title{
Primary Calvarial Tuberculosis (PCT) presenting as cold abscess of the scalp: A report of two cases
}

\author{
Vinod K.S. Gautam ${ }^{1}$, Sarbjeet Khurana ${ }^{2}$, Ravinder Singh ${ }^{3}$ \\ ${ }^{1}$ Department of Neurosurgery, ${ }^{2}$ Epidemiology, ${ }^{3}$ Medical Anthropology \\ Institute of Human Behaviour \& Allied Sciences, Dilshad garden, Delhi, India
}

\begin{abstract}
Calvarial tuberculosis is a rare manifestation of extra pulmonary tuberculosis. Primary calvarial tuberculosis, with no evidence tuberculosis elsewhere in the body is a rarer entity. We discuss the clinical presentation and management of two such patients subjected to investigations for any evidence of tuberculosis elsewhere in the body. Radiological images including CT scan and MRI of the brain were utilized. Histopathological examination of the granulation tissue and lytic bone established this diagnosis. A prompt surgery and anti tubercular therapy planned for patients and improved after a year. Early diagnosis and appropriate management plan of calvarial tuberculosis require high index of suspicion and knowledge. Radiological investigations help in diagnosis and preoperative planning in such cases. Definitive diagnosis is established with demostration of acid fast bacilli (AFB), culture and sensitivity, and histopathological examination. Prompt surgery and antitubercular therapy are the treatment of choice .
\end{abstract}

Keywords: Calvarium, tuberculosis, anti tubercular therapy

\section{INTRODUCTION}

Calvarial tuberculosis is a rare manifestation of extra pulmonary tuberculosis even in endemic regions [1]. Primary calvarial tuberculosis (PCT), with no evidence tuberculosis elsewhere in the body is a rarer entity [2]. Reid (1842) reported the earliest of flat skull bones tuberculosis in the literature [1-3]. We are to apprise the readers that the calvarial tuberculosis may present primarily with the scalp swelling without any other evidence of tuberculosis elsewhere in the body. So, there should be strong clinical suspicion and knowledge about this condition especially in a patient coming from endemic region of tuberculosis.

\section{MATERIAL AND MethodS}

A retrospective analysis of two patients' case records of pathologically verified calvarial tuberculosis treated in our tertiary care hospital. Clinical features, radiological findings, medical and surgical management, and outcomes were reviewed. Both patients were screened for the evidence of primary pulmonary tuberculosis. Images of skull X-ray, CT scan-head or MRI-brain or all were examined to assess the extent of brain, calvarium and subgaleal involvement and one of the authors (VKSG) surgically managed both patients. The histopathological findings were consistent with the diagnosis of tuberculosis, and then ATT was prescribed for a period of 12 months. We observed that both patients had completely recovered on follow-up after one year. Following is the brief of both the cases.

\section{Case 1}

A 12-year-old school girl from a middle class socioeconomic status family presented in the Neurosurgery OPD with a progressively increasing swelling over the left parietal region, noticed incidentally about 3 months back. There was no history of headache or any other constitutional symptoms of tuberculosis. The swelling was, $6 \mathrm{~cm} \times 5 \mathrm{~cm}$ in size; non-tender and with no signs of inflammation. There was a palpable calvarial defect. General physical and routine hematological examination did not reveal any abnormality except the erythrocyte sedimentation rate (ESR) was $40 \mathrm{~mm}$ in the first hour. X-ray of the skull revealed a lytic area in the left parietal bone. Computerized tomogram (Fig. 1) and MRI of the head (Fig. 2) showed extradural lesion in the left parietal lobe with destruction of the calvarium and subgaleal collection. Four drug regimen of antitubercular therapy was started consisting of Isoniazid, Rifampicin, Ethambutol and Pyrizinamide. Surgical evacuation of the granulation tissue and the debridement of the involved bone with evacuation of the extradural lesion was done under general anestheasia. Further, the pus, osteomyelitic bone and granulation tissue were sent for AFB staining and histopathological examination which had confirmed the diagnosis. Patients recovered completely after one year of antitubercular therapy. 


\section{Case 2}

A pregnant woman, 21 year, presented with a right frontal region progressively enlarging swelling for last 4 months. She also had occasional complaints of mild to moderate grade fever for last 1 month. There was no history of trauma. A swelling of $3 \mathrm{~cm} \mathrm{X} 3 \mathrm{~cm}$ in size, non-tender, with a palpable bony defect was noticed right frontal region. Further, the cranial X-ray films revealed osteomyelitis of right frontal bone and MRI of brain (Fig.3 and Fig.4) showed bony destruction with subgaleal collection with intracranial epidural extension. Patient underwent surgery under general anesthesia with excision of granulation tissue with craniectomy of involved osteomyelitic bone and evacuation of subgaleal collection. AFB staining and histopathogical examination confirmed the diagnosis of tuberculosis. Patient recovered completely following neurosurgery and one year course of anti tuberculous chemotherapy (Fig.5).

\section{FIGURES}

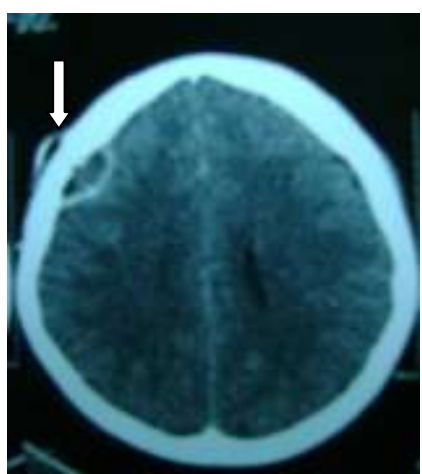

Figure-1. Case-1, Contrast enhanced computerized tomography of the skull showing osteomyelitis of the calvarial bone and hypodense peripherally enhancing extradural lesion

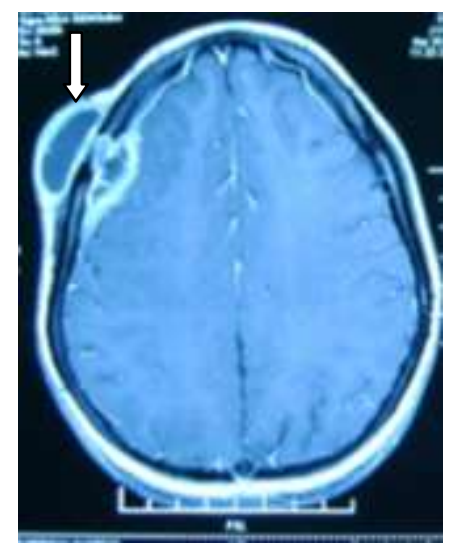

Figure 2. Case-1, Contrast MRI T1W1 axial image of brain showing subgaleal and extradural peripherally enhancing hypointense lesion

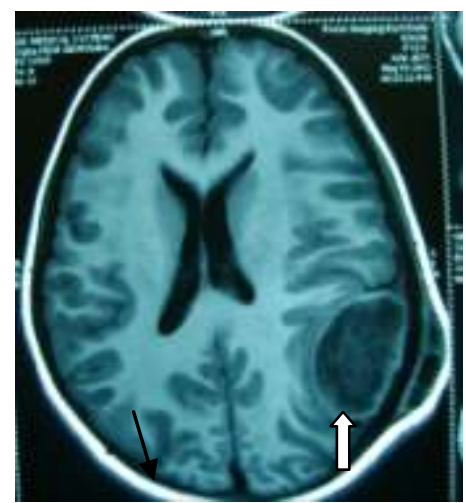

Figure 3. Case-2, MRI T1W1 axial image of brain showing subgaleal and extradural peripherally enhancing hypointense lesion 


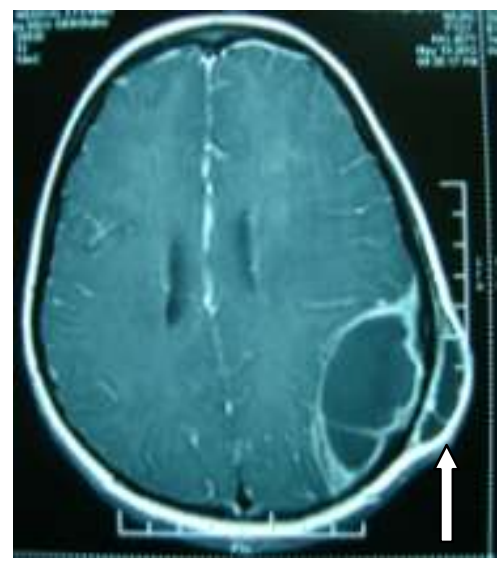

Figure 4. Case-2, Contrast MRI T1W1 axial image of brain showing subgaleal and extradural peripherally enhancing hypointen se lesion

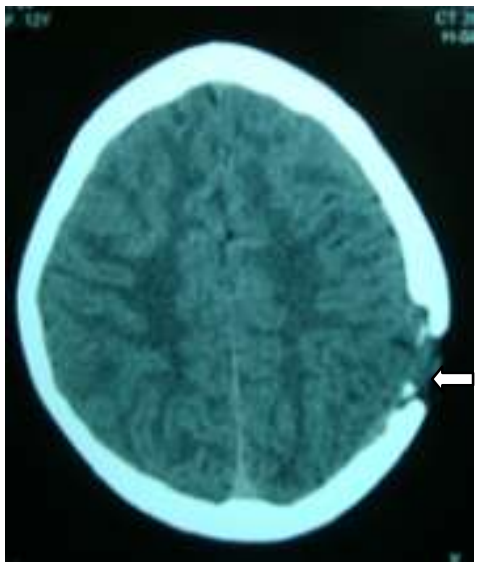

Figure 5. Case-2, Post operative CT scan of the patient showing complete resolution of the lesion after surgery and a course of ATT

\section{RESULT}

Both patients were females. The duration of symptoms was 3to 4 months. Both patients improved following the completion of therapy with no evidence of recurrence at end of one year follow up. The presenting features were scalp swelling without discharging sinus or pain. Computed tomography (CT) scan of brain showed lytic lesion in the calvarium with a peripherally enhancing extradural collection in both cases. The frontal bone was affected site of the calvarium in Case 1 and in Case 2 it was the parietal bone. CT scan and MRI brain depicted bony destruction, extradural granulation tissue and subgaleal collection in both cases. Both patients underwent surgical excision of necrotic bone and granulation tissue with primary closure of the scalp flap. Both patients showed complete clinical and radiological recovery following surgery and anti-tubercular therapy.

\section{DISCUSSION}

Calvarial tuberculosis is uncommon even in areas where tuberculosis is endemic [2]. It was first reported by Reid in 1842 and accounts for 0.2 to 1.3 of all tubercular osteitis [1,4]. After the advent of anti tubercular drug, the incidence has been reduced further and with the treatment of primary lesion, the calvarial lesion may not reach the stage of clinical presentation.

The younger population is at higher risk and it is prevalent equally in both sexes. In many cases the primary site of infection is elsewhere in the body, and the lungs are the most common site [2]. The tubercular process spreads to the calvarial bones through the blood stream [2,4], unlike lymphatic spread commonly seen in other bones. Concentrically placed proliferating fibroblasts encircle the tubercular granulation tissue and prevent its extension through the diploe. If the process is not contained, extension takes place through either of the tables. X-ray images may reveal three types of lesions, depending on the extent of bony involvement. Volkmann [6] used the designation perforating tuberculosis of the cranium for small, circumscribed, punchedout lesions in the bone, with granulation tissue covering both the inner and outer surfaces and with little tendency of the ostitis to spread in the bone or periosteum surrounding the defect. These lesions are commonly 
known as circumscribed lytic lesions. Konig [6] used the designation diffuse tuberculosis of the cranium for lesions involving widespread destruction of the inner table of the cranium, together with abundant extradural granulation tissue; these have been redefined as spreading-type lesions. The third and least common lesion type is the circumscribed sclerotic type[7].

CT scans reveal extradural compression, if present, and help to rule out associated intradural lesions as tuberculomas or subdural empyemas. CT scans with bone windows reveal the extent of bone involvement ${ }^{6}$. The demonstration of acid-fast bacilli (AFB) in pus smears, using the Ziehl-Neelsen stain, or the isolation of mycobacteria from cultures is a diagnostic criterion [9]. Microscopic examinations usually indicate infiltration with lymphocytes, plasma cells, and focal polymorphonuclear cells, with proliferating blood vessels [9]. Multiple epithelioid granulomas with Langhans-type giant cells can be observed with the necrotic material [3]. Surgery is indicated for cases involving large extradural collections causing neurological deficits, associated sinus formation, large pockets of caseating material, and intracranial extensions [8]. Extradural granulation tissue and the involved bone should be excised until normal bone is encountered. The dura should be left undisturbed. The sinus should be excised, with primary skin closure. Patients should be immediately treated with antituberculous chemotherapy. The response to the drugs should be monitored by clinical examinations, ESR, and CT scanning. Radiological evidence of the healing is seen after about two months of chemotherapy, with new bone formation at the edges of the lesion [9].

\section{CONCLUSION}

Calvarial tuberculosis is a rare presentation of the disease. Scalp swelling is the common presentation [8]. CT-head and MRI-brain are the main radiological investigations. However definitive diagnosis depends upon demonstration of acid-fast bacilli, culture and a biopsy report. Surgical debridement followed by antitubercular therapy for 12 months is the mainstay of the management of this unusual presentation. We feel that calvarial tuberculosis require high index of suspicion and knowledge for early diagnosis and proper management.

\section{REFERENCES}

[1] Strauss DC. Tuberculosis of flat bones of the vault of the skull. Surgical Gynecology and Obstetrics, 1933;57:384-398.

[2] Barton CJ. Tuberculosis of the vault of the skull. British Journal of Radiology 1961; 34:286-290.

[3] Jadhav RN, Palande DA. Calvarial tuberculosis. Neurosurgery 1999; 45:1345-50.

[4] Meng CM, WU YK. Tuberculosis of the flat bones of the vault of the skull. Journal of Bone Joint Surgery 1942; 34:341-353.

[5] Mohanty S, Rao CJ, Mukherjee KC. Tuberculosis of the skull. International Surgery. 1981; 66:81-83

[6] Batuk Diyora, Rajiv Kumar, Rahul Modgi, Alok Sharma: A report of eleven patients. Neurology India, Sep-Oct 2009, Vol 57, issue 5, 607-612.

[7] Gupta, P K; Kolluri, V. R., Chandramouli B.A, Venkataramana, N.K., Das B. Calvarial tuberculosis: A report of two cases. Neurosurgery, 1989; 25:830-3.

[8] Le Roux PD, Griffin GE, Marsh HT, Winn HR. Tuberculosis of the skull; A rare condition-Case report and review of the literature. Neurosurgery, 1990; 26:851-5.

[9] Tyagi AK, Kirollos RW, Kang NV. Tuberculous osteomyelitis of the skull: A case report and review of the literature. British Journal Neurosurgery. 1996; 10:399-401. 